\title{
Epitope Presentation on Monolayer-Protected Clusters for Multi-component Functional Nanostructures
}

\author{
Aren E. Gerdon, David W. Wright*, and David E. Cliffel*
}

Department of Chemistry, Vanderbilt University, VU Station B, Nashville, Tennessee 37235-1822

S-1. Title and Table of Contents

S-2. TEM image of Tiop-MPC, showing spherical cluster

S-3. ${ }^{1}$ H NMR of Tiop-MPC

S-4. HPLC trace of HA peptide

S-5. MALDI mass spectrum of HA peptide

S-6. ${ }^{1} \mathrm{H}$ NMR functionalized HA-MPC 


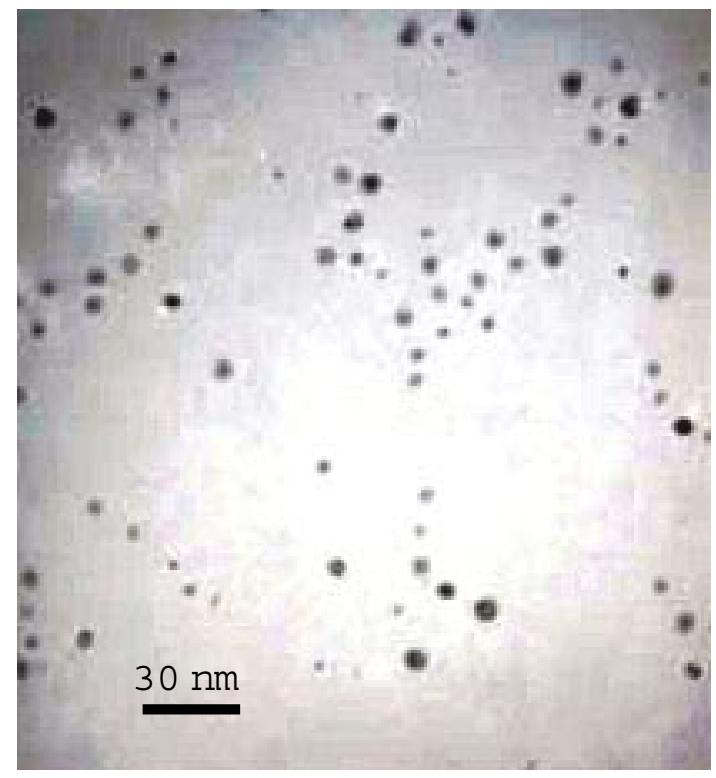

S-2. TEM image of Tiop-MPC, showing spherical cluster. 

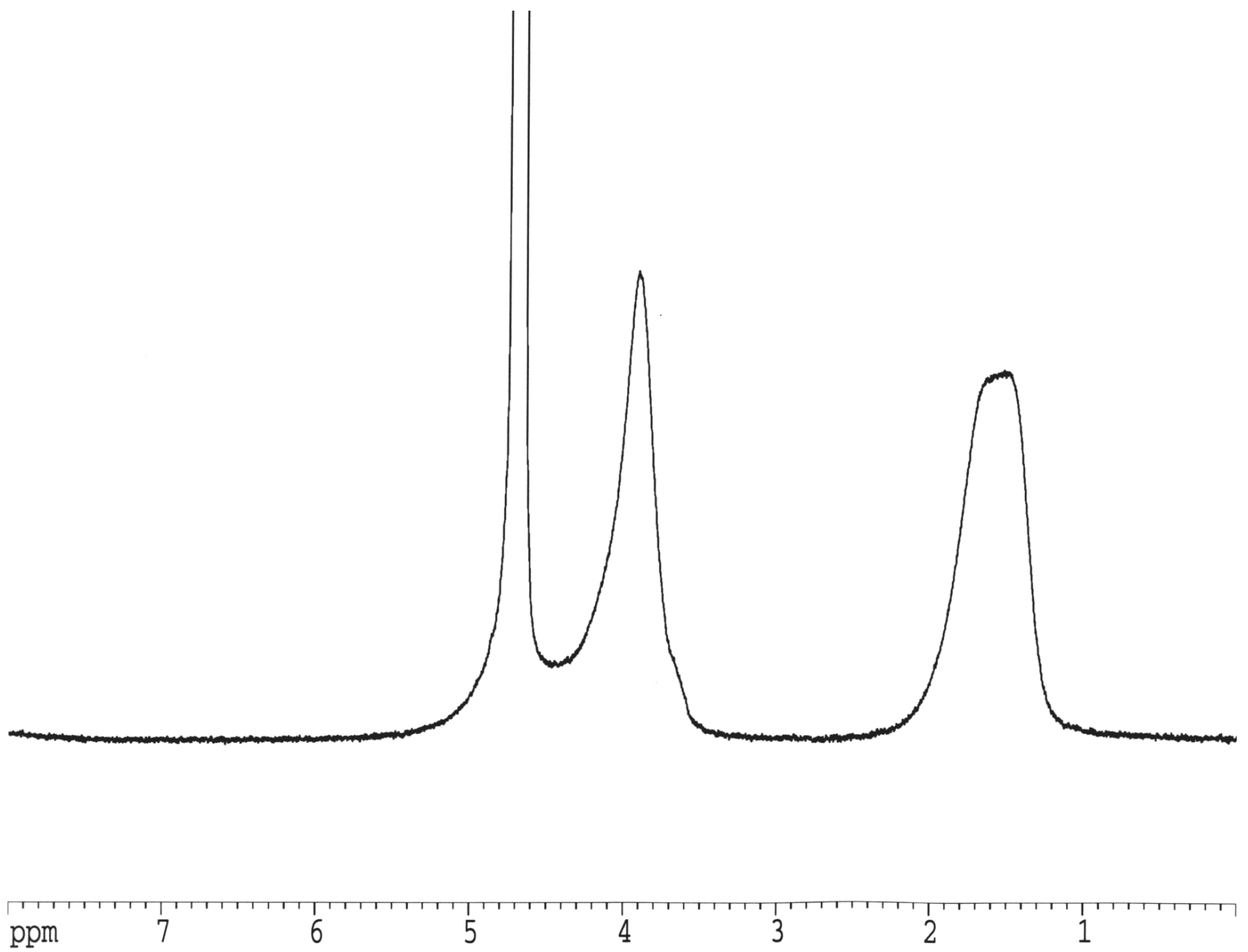

S-3. ${ }^{1}$ H NMR of Tiopronin MPC. Broad peaks are indicative of nanocluster formation. A lack of sharp peaks indicates purity. 


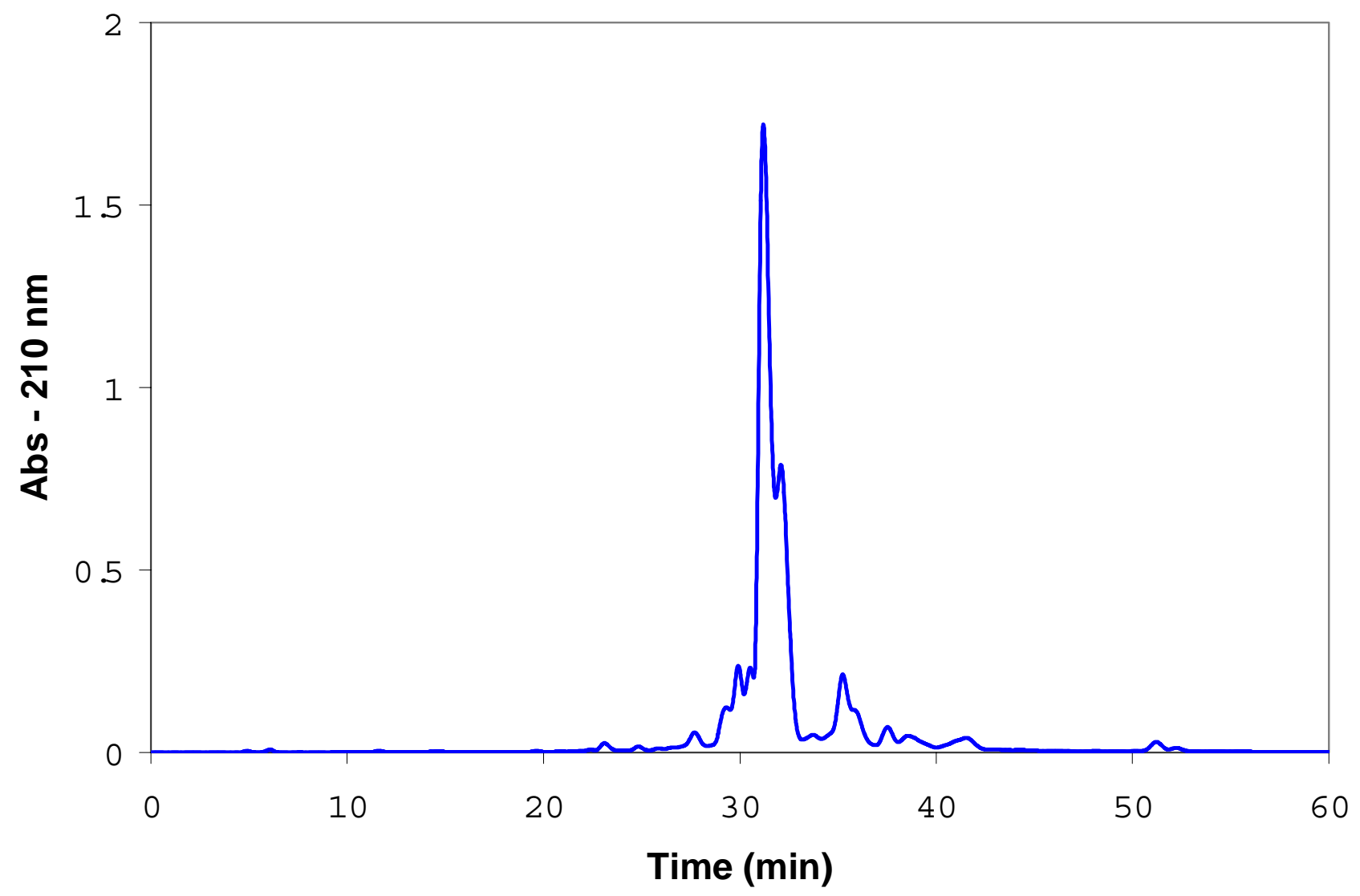

S-4. Chromatogram from the purification of the crude HA peptide using semi-preparative HPLC. The relatively sharp peak and lack of other major peaks indicates a homogenous sample. 


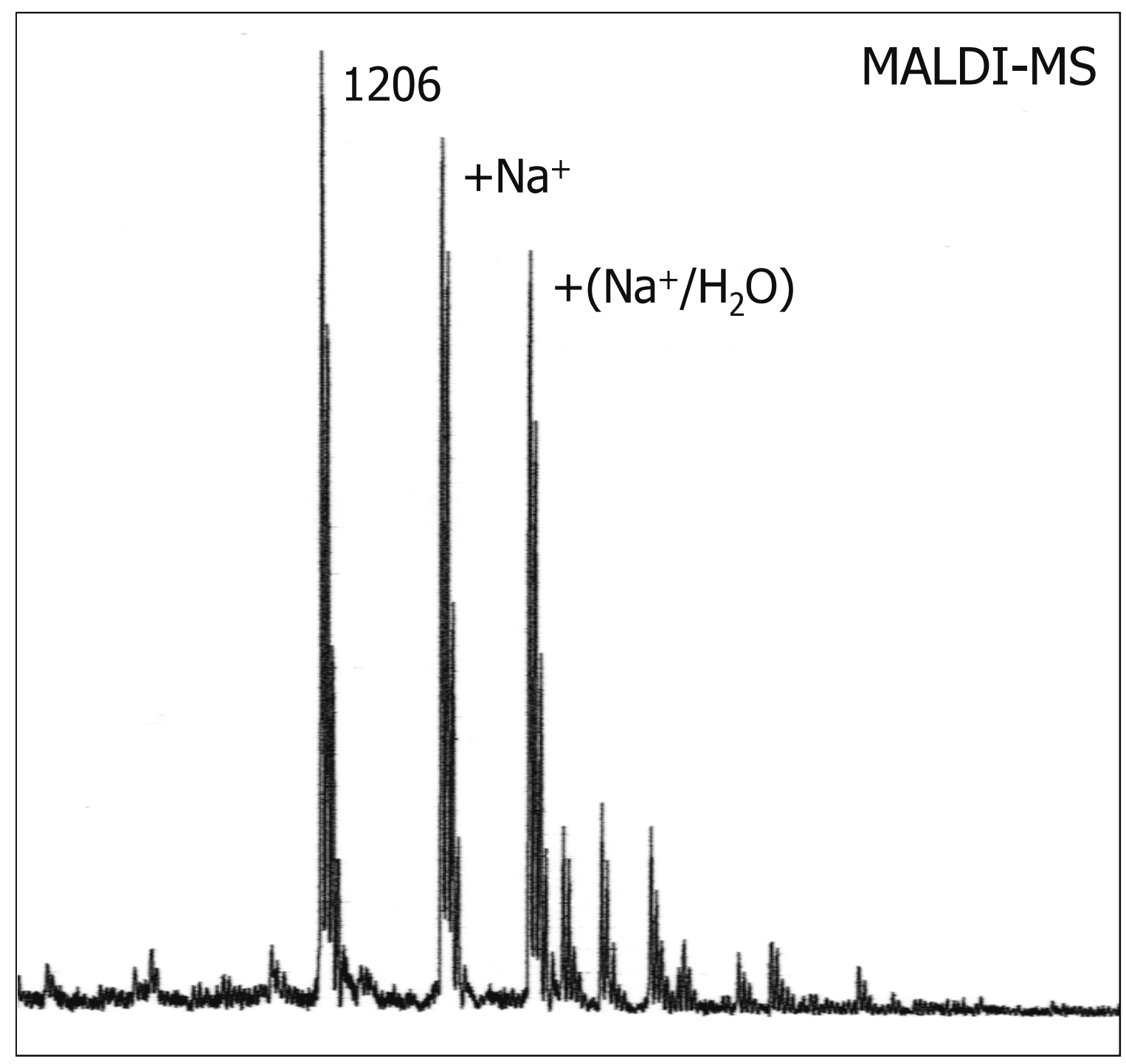

S-5. MALDI-MS of HA peptide collected from the major peak of HPLC (S-4). Major peak (1206) is indicative of the pure peptide $+\mathrm{H}^{+}$. Other peaks are listed according to their adducts. 


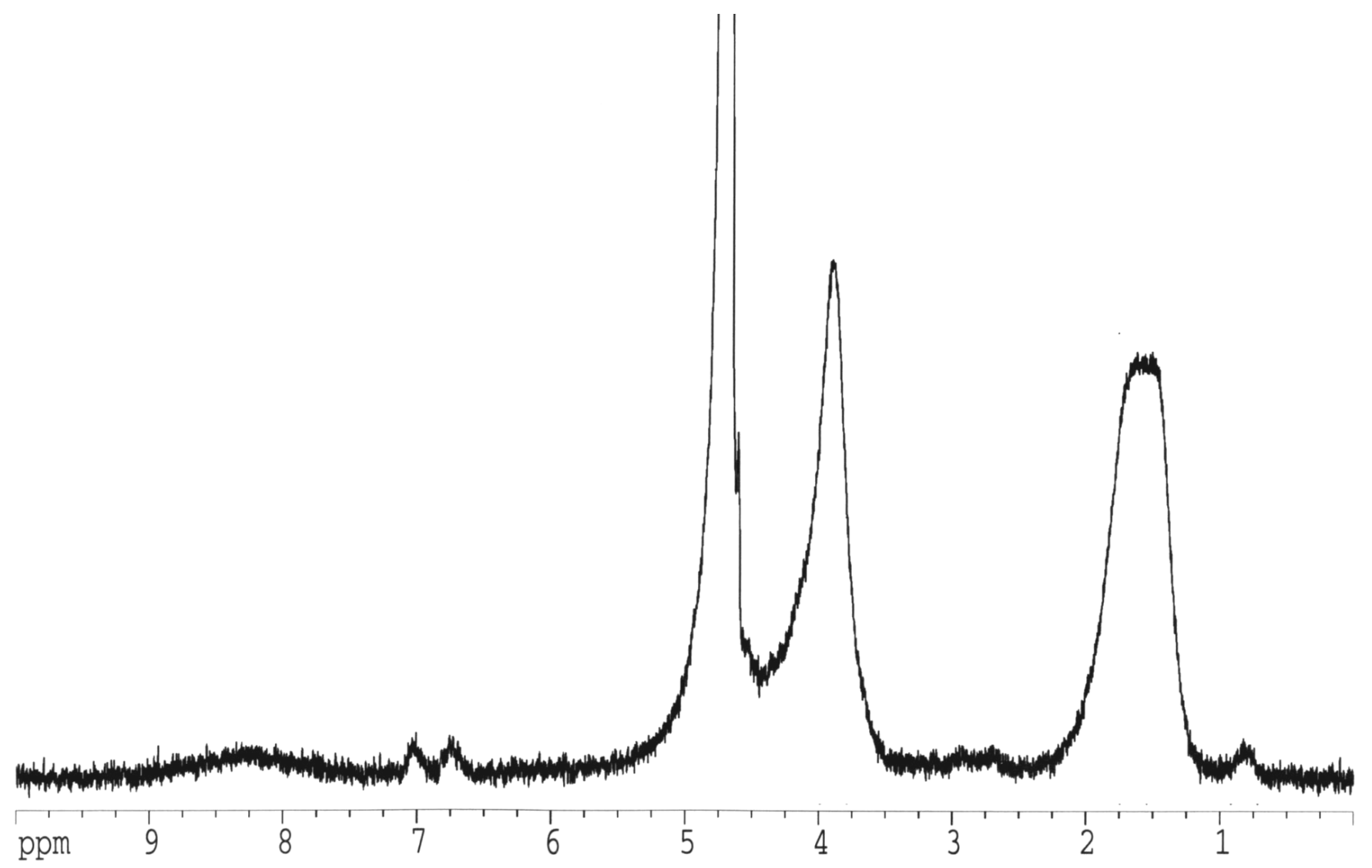

S-6. ${ }^{1} \mathrm{H}$ NMR of tiopronin-MPC after place exchange with HA peptide epitope. Tiopronin peaks are still present. Peptide peaks are visible at approximately $0.8,2.8,6.8$, and $7.0 \mathrm{ppm}$. The number of peptides per cluster is determined through NMR integration values and the following equation:

$[\text { Normalized Peak Area }]_{\text {Tiop }} /[\text { H per Ligand }]_{\text {Tiop }}:[\text { Normalized Peak Area }]_{\text {peptide }} /[\text { H per ligand }]_{\text {peptide }}$ 